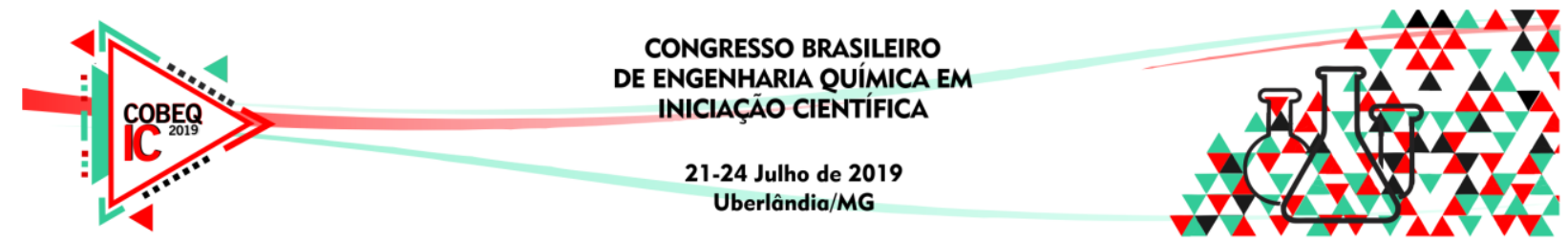

\title{
IMPLEMENTAÇÃO DE INTERPOLADORES MULTILINEARES COMO MODELOS SIMPLIFICADOS DE REAÇÕES CATALISADAS POR LIPASES.
}

\author{
W. M. SANTOS ${ }^{1}$, M. P.A. RIBEIRO ${ }^{1,2}$ e R. C. GIORDANO ${ }^{1,2}$ \\ ${ }^{1}$ Universidade Federal de São Carlos, Departamento de Engenharia Química \\ ${ }^{2}$ Pós-Graduação em Engenharia Química, Universidade Federal de São Carlos (PPG-EQ / \\ UFSCar) \\ E-mail para contato: marceloribeiro@ufscar.br
}

\begin{abstract}
RESUMO - Modelos dinâmicos completos de reações enzimáticas são flexíveis e podem ser usados para simular e otimizar reações em batelada e batelada alimentada, modos de operação comuns em reações enzimáticas. Contudo, incorporar modelos dinâmicos complexos em simuladores de processo para descrever uma planta inteira pode aumentar muito o esforço computacional. Isso pode dificultar o uso desses simuladores para otimizar a planta como um todo. Este trabalho, tem como objetivo a criação e a avaliação de modelos simplificados (interpoladores multilineares) de reações enzimáticas catalisados por lipases. Esses modelos simplificados serão utilizados posteriormente, na análise tecnoeconômica de uma biorrefinaria integrada biodiesel-bioetanol. O modelo simplificado foi baseado em modelo cinético de síntese enzimática de biodiesel etílico encontrado na literatura. Avaliou-se o erro obtido no modelo simplificado em relação ao modelo completo em função do número de pontos de discretização das variáveis de entrada. Resultados mostraram que 90 pontos de discretização foram o suficiente para reduzir o erro do modelo simplificado em menos que $1 \%$.
\end{abstract}

\section{INTRODUÇÃO}

Uma biorrefinaria integrada biodiesel-bioetanol pode ser uma maneira de reduzir os custos de produção desses biocombustíveis. Isso ocorre pela otimização das utilidades desses dois processos e pelo entrecruzamento de correntes como, por exemplo, o uso do etanol como álcool para a transesterificação do biodiesel e do uso do glicerol, resíduo da produção do biodiesel, na coluna de desidratação do etanol anidro. Para se chegar a uma condição otimizada da biorrefinaria integrada, simuladores de processos são ferramentas importantes, assim como, modelos dos mais importantes reatores.

Geralmente, reação transesterificação é uma seqüência de três etapas consecutivas, de reações reversíveis. Na primeira, a partir dos triglicerídeos, obtêm-se os diglicerídeos. A partir dos diglicerídeos, são produzidos os monoglicerídeos, e na última etapa os monoglicerídeos dão origem à glicerina, levando em conta que em todas as etapas são produzidos ésteres; ver Figura 1. A relação estequiométrica entre álcool e óleo é de 3:1 como mostra na Figura 2, 


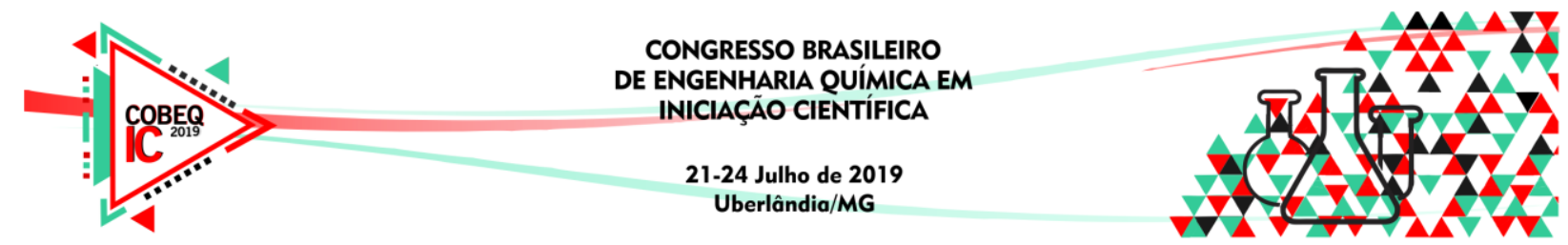

pelo qual um excesso de álcool é usualmente usado para aumentar a reação em direção aos produtos desejados. A razão molar entre o álcool e o óleo vegetal é a variável mais importante da reação de transesterificação. De acordo com o princípio de Le Chatelier, um excesso de álcool aumenta a conversão em éster porque desloca o equilíbrio para o lado direito da reação (FILLIÈRES, 1995). A utilização de grande excesso de álcool provoca a formação de uma fase líquida, homogênea, no final da reação. Esse fato acontece porque o excesso de álcool solubiliza a glicerina, provocando, conseqüentemente, uma diminuição da densidade da glicerina impedindo a precipitação (DE LIMA, 2006).

Figura 1. Etapas da reação de transesterificação

$$
\begin{gathered}
\text { TRIGLICERIDEOS }(T G)+R O H \underset{K 1, K 2}{\longrightarrow} \text { DIGLICERIDEOS }(D G)+\text { RCOOR }_{1} \\
\text { DIGLICERIDEOS }(D G)+R O H \underset{K 3, K 4}{\longleftrightarrow} \text { MONOGLICERIDEOS }(M G)+\text { RCOOR }_{2} \\
\text { MONOGLICERIDEOS }(M G)+R O H \underset{K 5, K 6}{\longrightarrow} \text { GLICERINA }(G L)+\text { RCOOR }_{3}
\end{gathered}
$$

Figura 2. Equação química geral da reação de transesterificação de triglicerídeos

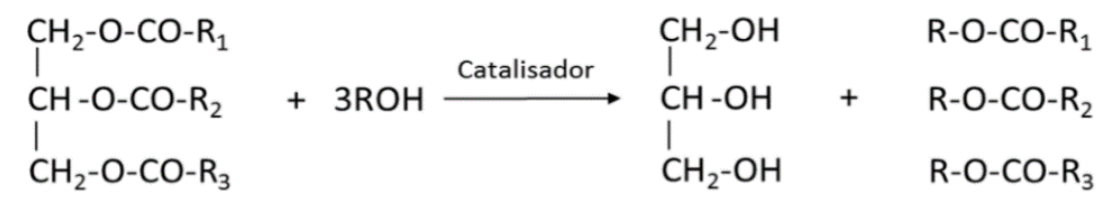

Esses modelos são importantes para a otimização do reator em si, pois podem ser utilizados para encontrar janelas importantes de condições de processo. Porém, embora esses modelos cinéticos possam ser utilizados dentro de um simulador da planta como um todo, eles são muito complexos. Observa-se que, do ponto de vista industrial, apenas algumas regiões de condições reacionais e de operação são realmente viáveis. Assim, modelos simplificados capazes de representar variáveis importantes de desempenho de processos (conversão, rendimento e produtividade) em regiões coerentes de condições de operação ajudam reduzir o esforço computacional para a otimização da biorrefinaria integrada.

Neste trabalho utilizou-se um modelo cinético de síntese enzimática de biodiesel encontrado na literatura para construir um modelo simplificado (interpolador multilinear) para o cálculo do rendimento de biodiesel, baseado na quantidade de óleo disponibilizada. Simulações da síntese enzimática em batelada foram realizadas variando-se a concentração inicial de óleo (razão óleo/etanol) e tempos finais de reação.

\section{MATERIAIS E MÉTODOS}

\subsection{Métodos}




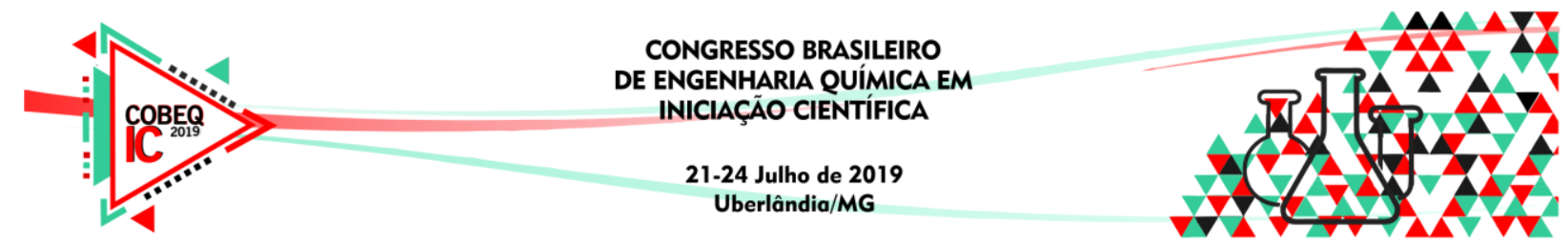

Implementação dos códigos: os algoritmos utilizados foram implementados no software Scilab 5.5.2. Algoritmo para o cálculo do Latin Hypercube Sampling é disponibilizado pelo pacote NISP dessa versão do Scilab.

Modelo cinético: O modelo proposto por Cheirsilp et al. (2008) para a transesterificação enzimática para a produção de biodiesel foi escolhido inicialmente como modelo base para a implementação do programa para a construção de interpolador multilinear. Como representado nas seguintes equações:

$$
\begin{aligned}
& \mathrm{d}[\mathrm{T}] / \mathrm{dt}=-\left(\mathrm{V}_{\mathrm{mT}}[\mathrm{W}]+\mathrm{V}_{\mathrm{eT}}[\mathrm{Al}]\right)[\mathrm{T}]\left[\mathrm{E}^{*}\right] \\
& \mathrm{d}[\mathrm{D}] / \mathrm{dt}=\left(\left(\mathrm{V}_{\mathrm{mT}}[\mathrm{W}]+\mathrm{V}_{\mathrm{eT}}[\mathrm{Al}]\right)[\mathrm{T}]-\left(\mathrm{V}_{\mathrm{mD}}[\mathrm{W}]+\mathrm{V}_{\mathrm{eD}}[\mathrm{Al}]\right)[\mathrm{D}]\right)\left[\mathrm{E}^{*}\right] \\
& \mathrm{d}[\mathrm{M}] / \mathrm{dt}=\left(\left(\mathrm{V}_{\mathrm{mD}}[\mathrm{W}]+\mathrm{V}_{\mathrm{eD}}[\mathrm{Al}]\right)[\mathrm{D}]-\left(\mathrm{V}_{\mathrm{mM}}[\mathrm{W}]+\mathrm{V}_{\mathrm{eM}}[\mathrm{Al}]\right)[\mathrm{M}]\right)\left[\mathrm{E}^{*}\right] \\
& \mathrm{d}[\mathrm{G}] / \mathrm{dt}=\left(\mathrm{V}_{\mathrm{mM}}[\mathrm{W}]+\mathrm{V}_{\mathrm{eM}}[\mathrm{Al}]\right)[\mathrm{M}]\left[\mathrm{E}^{*}\right] \\
& \mathrm{d}[\mathrm{F}] / \mathrm{dt}=\left(\left(\mathrm{V}_{\mathrm{mT}}[\mathrm{T}]+\mathrm{V}_{\mathrm{mD}}[\mathrm{D}]+\mathrm{V}_{\mathrm{mM}}[\mathrm{M}]\right)[\mathrm{W}]-\mathrm{V}_{\mathrm{eEs}}[\mathrm{F}][\mathrm{Al}]\right)\left[\mathrm{E}^{*}\right] \\
& \mathrm{d}[\mathrm{W}] / \mathrm{dt}=-\left(\mathrm{V}_{\mathrm{mT}}[\mathrm{T}]+\mathrm{V}_{\mathrm{mD}}[\mathrm{D}]+\mathrm{V}_{\mathrm{mM}}[\mathrm{M}]\right)[\mathrm{W}]\left[\mathrm{E}^{*}\right] \\
& \mathrm{d}[\mathrm{Es}] / \mathrm{dt}=-\mathrm{d}[\mathrm{Al}] \mathrm{dt}=\left(\mathrm{V}_{\mathrm{eT}}[\mathrm{T}]+\mathrm{V}_{\mathrm{eD}}[\mathrm{D}]+\mathrm{V}_{\mathrm{eM}}[\mathrm{M}]+\mathrm{V}_{\mathrm{eEs}}[\mathrm{F}]\right)[\mathrm{Al}]\left[\mathrm{E}^{*}\right] \\
& {\left[\mathrm{E}^{*}\right]=\left[\mathrm{E}_{\mathrm{T}}\right] /\left(1+\mathrm{Km}_{\mathrm{T}}[\mathrm{T}]+\mathrm{Km} \mathrm{m}_{\mathrm{D}}[\mathrm{D}]+\mathrm{Km}_{\mathrm{M}}[\mathrm{M}]+\mathrm{Km}_{\mathrm{F}}[\mathrm{F}]+\left([\mathrm{Al}] / \mathrm{K}_{\mathrm{I}}\right)\right)}
\end{aligned}
$$

Onde: As taxas de aparecimento de intermediários hidrolisados incluindo triacilglicerol [T], diacilglicerol [D], monoacilglicerol [M] e glicerol [G] são expressos nas equações (1)-(4). As taxas de aparecimento de ácido graxo livre [F], água [W], éster etílico de ácido graxo (Es) e etanol [Al] das etapas de hidrólise e esterificação são apresentadas nas Eqs (5)-(7). [ $\left.\mathrm{E}_{\mathrm{T}}\right]$ é a concentração total de enzima alimentada no reator. $\mathrm{V}_{\mathrm{mT}}, \mathrm{V}_{\mathrm{mD}}, \mathrm{V}_{\mathrm{mM}}, \mathrm{V}_{\mathrm{eEs}}, \mathrm{V}_{\mathrm{eT}}, \mathrm{V}_{\mathrm{eD}}$ and $\mathrm{V}_{\mathrm{eM}}$ são constantes de velocidade cujos valores são $7,619 \times 10^{-2}, 8,128 \times 10^{-2}, 1,951 \times 10^{-1}, 1,383$, 2,751, 1,176 e 0,965 $\mathrm{mmol}^{-1} \mathrm{~h}^{-1}$, respectivamente. Enquanto, $\mathrm{Km}_{\mathrm{T}}, \mathrm{Km}_{\mathrm{D}}, \mathrm{Km}_{\mathrm{M}}$ e $\mathrm{Km}_{\mathrm{F}}$ são constantes de equilíbrio com valores de $2,891 \times 10^{-2}, 2,322 \times 10^{-2}, 1,974 \times 10^{-2}$ e $1,121 \times 10^{-2}$ $\mathrm{mmol}^{-1} \mathrm{~h}^{-1}$, respectivamente. A constante de inibição $\left(\mathrm{K}_{\mathrm{I}}\right)$ foi de $0,883 \mathrm{mmol}^{-1} \mathrm{~h}^{-1}$.

Condições de processo simulado: Os dados de entrada (variáveis independentes) foram a concentração inicial de óleo (variando de 0,1 a 2,0 mmol/g), e tempo de reação (variando de 1 a $10 \mathrm{~h}$ ), mantidas as concentrações de etanol, água e enzima, constantes, iguais a 0,28; 0,5 e $0,2 \mathrm{mmol} / \mathrm{g}$, respectivamente. A variável resposta (variável dependente) calculada foi o rendimento em biodiesel.

Construção do modelo multilinear: o número de pontos de discretização foi de 2 a 100 para cada variável independente variada. A partir do resultado da simulação utilizando o modelo cinético completo, construiu-se um modelo de interpolação linear para o rendimento do processo baseado nas variáveis de entrada.

Avaliação do erro: Para avaliar o erro do modelo simplificado frente ao modelo completo, utilizou-se o Latin hypercube sampling para amostrar pontos aleatórios de forma homogênea em toda a região utilizada das variáveis de entrada. O número de pontos amostrados foi de 10 vezes o número de regiões obtidas pelos pontos de discretização.

\section{RESULTADOS E DISCUSSÃO}

A Figura 3 mostra os resultados obtidos para o estudo de caso. Observa-se claramente na Fig.3(esq.) que com o aumento do número de pontos de interpolação, o erro relativo no cálculo do rendimento se reduz. Observou-se também que ambas as variáveis independentes avaliadas têm não-linearidades parecidas frente ao cálculo do rendimento, pois o aumento no 


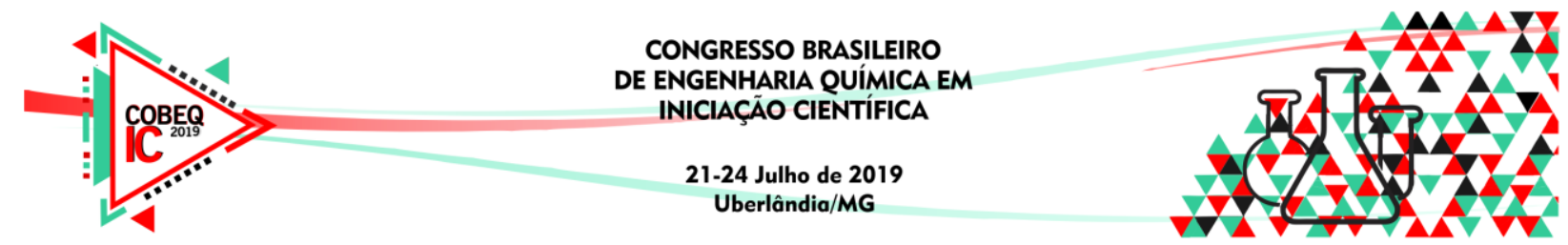

número de pontos discretos das duas variáveis reduz de forma parecida o erro do modelo. Nem sempre é esse o caso. É possível que o aumento na discretização de uma variável, a partir de certo ponto, não melhore o modelo, porém o aumento de outra sim.

Os resultados mostram também que, para que haja um desvio padrão do erro relativo (entre o modelo completo e o simplificado) menor que $1 \%$, são necessários pelo menos 90 pontos total de discretização (malha $9 \times 10$ ). A Figura 3 (direita) mostra o rendimento calculado pelo modelo simples versus o dado pelo modelo completo, para essa malha. Sendo $\mathrm{x}_{1}$ e $\mathrm{x}_{2}$ a concentração de óleo e o tempo de reação, respectivamente.

Figura 3 - Esquerda: Número de pontos de discretização (total, concentração inicial de óleo = $\mathrm{x}_{1}$ e tempo de reação $=\mathrm{x}_{2}$ ) versus log do desvio padrão do erro relativo no cálculo do rendimento em biodiesel. Direita: Rendimento dado pelo modelo simplificado versus o dado pelo modelo completo para a malha $9 \times 10$.
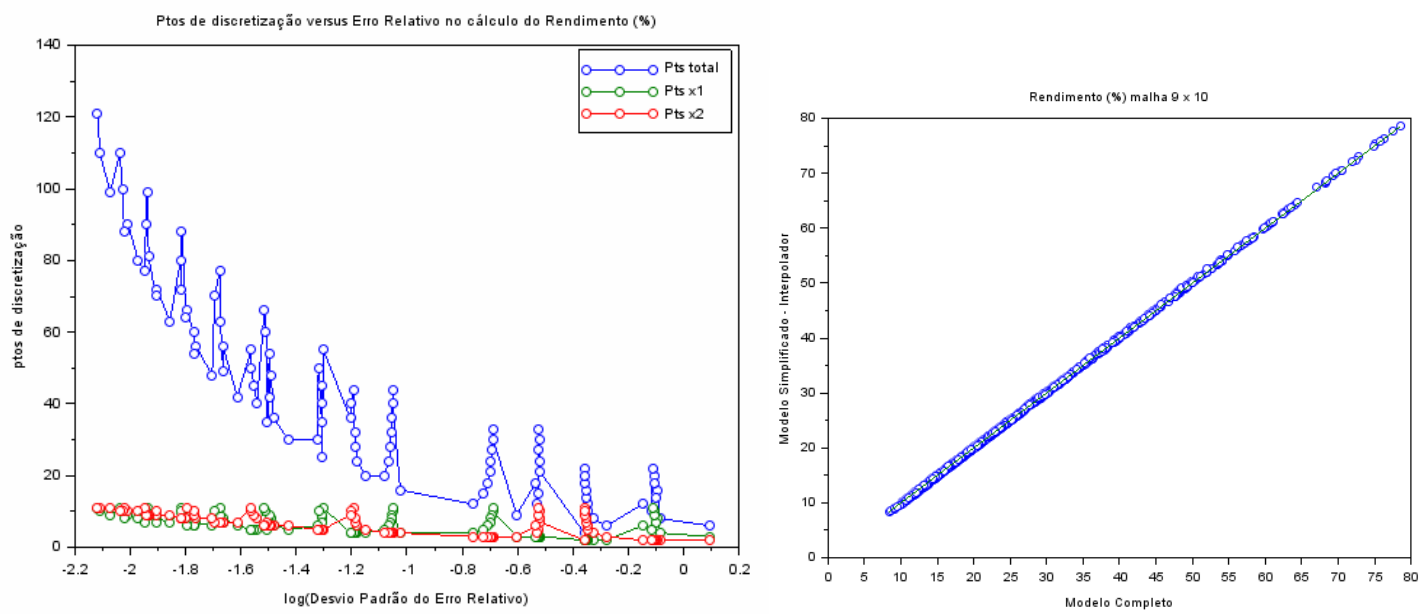

\section{CONCLUSÃO}

Os resultados obtidos mostraram que o interpolador multilinear é capaz de aproximar o modelo dinâmico com um desvio padrão do erro relativo menor que $1 \%$ quando se utiliza uma malha de 90 pontos. Outras variáveis independentes como a concentração de enzima e água podem ser incorporadas no modelo. Além disso, outras variáveis dependentes como a conversão do álcool e a seletividade transesterificação/hidrólise poderão utilizadas como respostas do modelo. Esses modelos simplificados serão integrados posteriormente em simulação de uma biorrefinaria utilizando o software EMSO.

\section{AGRADECIMENTOS}

Este trabalho foi apoiado pelo processo no 2018/18586-5, Fundação de Amparo à Pesquisa do Estado de São Paulo (FAPESP). As opiniões, hipóteses e conclusões ou 


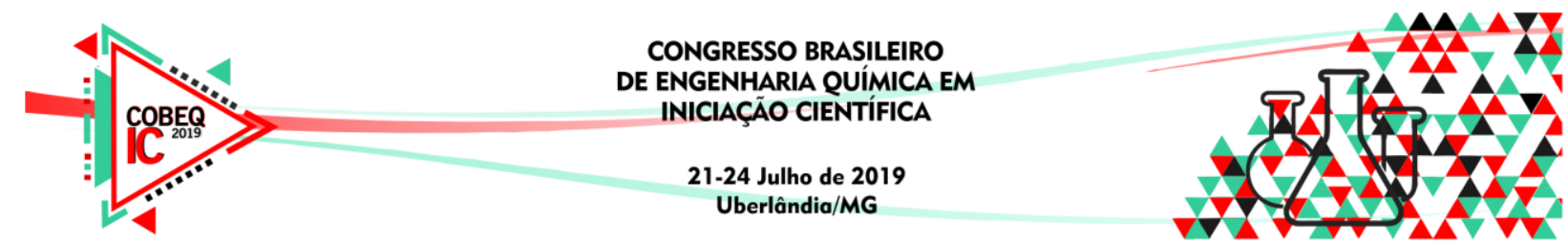

recomendações expressas neste material são de responsabilidade dos autores e não necessariamente refletem a visão da FAPESP.

\section{REFERÊNCIAS BIBLIOGRÁFICAS}

CHULALAKSANANUKUL S, LONGO MA, CHULALAKSANANUKUL W, CONDORET JS, COMBES D. Lipase-catalysed esterification reaction in an organic solvent: comparison between free and immobilised biocatalysts. Afinidad. 1999;56:121-125.

CYGLER, M.; SCHRAG, J. D. Structure as basis for understanding interfacial properties of lipases. Methods in enzymology Lipases, Part A: Biotechnology, New York, v. 284, p. 3-27, 1997.

CHEIRSILP, B. et al. Impact of transesterification mechanisms on the kinetic modeling of biodiesel production by immobilized lipase. Biochemical Engineering Journal, 2008, 42, 261-269.

DE LIMA N.,Otimização das variáveis de processo da transesterificação (etanólise) do óleo de mamoma: Produção de biodiesel, Faculdade de Engenharia Química, UNICAMP, 2006

FERNANDEZ-LAFUENTE, R. Review: Lipase from Rhizomucor miehei as biocatalyst in fats and oils modification. Journal of Molecular Catalysis B: Enzymatic, v. 66, p. 15-32, 2010a.

FILLÈRES R., BENJELLOUM-MLAYAH B. E DELMAS M., Ethanolysis of rapeseed oil: quantitation of ethyl esters, mono-, di-, and triglycerides and glycerol by highperformance size-exclusion chromatography, Journal of American Oil Chemists' Society, volume $72, \mathrm{n}^{\circ} .4,1995$.

VULFSON, E. N. Alteration of lipase chain length specificity in the hydrolysis of esters by random mutagenesis. Biotechnology and Bioengineering, v. 73, p. 433-441, 2001. 